\title{
Erratum: Magnon-bipolar carrier drag thermopower in antiferromagnetic/ferromagnetic semiconductors: Theoretical formulation and experimental evidence [Phys. Rev. B 102, 045202 (2020)]
}

Md Mobarak Hossain Polash $₫$ and Daryoosh Vashaee ${ }^{(0)}$

(Received 1 January 2021; published 26 January 2021)

DOI: 10.1103/PhysRevB.103.039902

In Sec. II of the paper, following Eq. (4), a particular case was assumed where $\tau_{m} \gg \tau_{m e}$. This condition usually holds at sufficiently low temperature $T \ll T_{C}$. When the temperature approaches the transition temperature $T_{C}$, we note that, in many magnetic semiconductors, an opposite relation can hold, i.e., $\tau_{m} \ll \tau_{m e}$. Therefore, an expression for the total thermopower in a bipolar magnetic semiconductor is derived with the later condition and presented in this erratum.

Electrical conductivity for electrons considering the diffusion and drag contributions can be expressed as in Eq. (4) of the paper,

$$
\sigma_{n}=\tau_{m} \frac{e^{2} n}{m_{e}^{*}}\left(\frac{1}{\tau_{e 0}}+\frac{1}{\tau_{e m}}\right)^{-1}\left(\frac{1}{\tau_{m}}+\frac{1}{\tau_{m e}}\right)
$$

Considering, $\tau_{e 0} \gg \tau_{e m}$ and $\tau_{m e} \gg \tau_{m}$, one can write

$$
\sigma_{n}=\frac{e^{2} n}{m_{e}^{*}} \tau_{e m}
$$

The expressions for $\sum_{n}^{\text {diff }}$ and $\sum_{n}^{\text {drag }}$ can be expressed as

$$
\begin{gathered}
\sum_{n}^{\mathrm{diff}}=-\frac{k_{B}}{e}\left[\left(\ln \frac{N_{c}}{n}\right)+\left(r+\frac{5}{2}\right)\right] \frac{e^{2} n}{m_{e}^{*}} \tau_{e m}\left(1+\frac{\tau_{m}}{\tau_{m e}}\right) \approx-\frac{e n k_{B}}{m_{e}^{*}} \tau_{e m}\left[\left(\ln \frac{N_{c}}{n}\right)+\left(r+\frac{5}{2}\right)\right], \\
\sum_{n}^{\mathrm{drag}}=-\frac{m_{e}^{*} c^{2}}{e T} \frac{\tau_{m}}{\tau_{e m}} \frac{\tau_{m e}}{\left(\tau_{m}+\tau_{m e}\right)} \frac{e^{2} n}{m_{e}^{*}} \tau_{e m}\left(1+\frac{\tau_{m}}{\tau_{m e}}\right) \approx-\tau_{m} \frac{e n c^{2}}{T},
\end{gathered}
$$

Similar expressions as (E2) and (E3) hold for the holes, where $n, \tau_{e m}$, and $N_{c}$ are replaced with $p$, $\tau_{h m}$, and $N_{v}$, respectively. Hence, the total diffusion contribution to the thermopower is as follows:

$$
\sum^{\mathrm{diff}}=\frac{e p k_{B}}{m_{h}^{*}} \tau_{h m}\left[\left(\ln \frac{N_{v}}{p}\right)+\left(r+\frac{5}{2}\right)\right]-\frac{e n k_{B}}{m_{e}^{*}} \tau_{e m}\left[\left(\ln \frac{N_{c}}{n}\right)+\left(r+\frac{5}{2}\right)\right] .
$$

Similarly, the total drag contribution to the thermopower can be written as

$$
\sum^{\mathrm{drag}}=\tau_{m} \frac{e p c^{2}}{T}-\tau_{m} \frac{e n c^{2}}{T}=\frac{e c^{2} \tau_{m}}{T}(p-n) .
$$

Adding (E5) and (E6), and inserting the expressions for $\sigma_{n}$ and $\sigma_{p}$, we have

$$
S=\frac{k_{B}}{e} \frac{\frac{p}{m_{h}^{*}} \tau_{h m}\left[\left(\ln \frac{N_{v}}{p}\right)+\left(r+\frac{5}{2}\right)\right]-\frac{n}{m_{e}^{*}} \tau_{e m}\left[\left(\ln \frac{N_{c}}{n}\right)+\left(r+\frac{5}{2}\right)\right]+\frac{c^{2}}{k_{B} T} \tau_{m}(p-n)}{\frac{p}{m_{h}^{*}} \tau_{h m}+\frac{n}{m_{e}^{*}} \tau_{e m}} .
$$

For a nondegenerate semiconductor, $\tau_{e m}$ can be written in terms of $\tau_{m e}$ according to

$$
\tau_{e m}=\frac{3 \pi^{1 / 2} n}{2 N_{c}} \tau_{m e} \frac{m_{e}^{*} c^{2}}{k_{B} T} .
$$


A similar expression holds for $\tau_{h m}$. Replacing $\tau_{e m}$ and $\tau_{h m}$ in (E7) with their equivalent expressions versus $\tau_{m e}$ and $\tau_{m h}$, we can write

$$
S=\frac{k_{B}}{e} \frac{\frac{p^{2}}{N_{v}} \tau_{m h}\left[\left(\ln \frac{N_{v}}{p}\right)+\left(r+\frac{5}{2}\right)\right]-\frac{n^{2}}{N_{c}} \tau_{m e}\left[\left(\ln \frac{N_{c}}{n}\right)+\left(r+\frac{5}{2}\right)\right]+\frac{2}{3 \sqrt{\pi}} \tau_{m}(p-n)}{\frac{p^{2}}{N_{v}} \tau_{m h}+\frac{n^{2}}{N_{c}} \tau_{m e}} .
$$

If $\tau_{m} \ll \tau_{m e}$ such that one can ignore $\frac{2}{3 \sqrt{\pi}} \tau_{m}(p-n)$ compared to $\frac{n^{2}}{N_{c}} \tau_{m e}\left[\left(\ln \frac{N_{c}}{n}\right)+\left(r+\frac{5}{2}\right)\right]$, and similarly for the holes, one can further simplify Eq. (E9) and arrive at

$$
S=\frac{k_{B}}{e} \frac{\frac{p^{2}}{N_{v}} \tau_{m h}\left[\left(\ln \frac{N_{v}}{p}\right)+\left(r+\frac{5}{2}\right)\right]-\frac{n^{2}}{N_{c}} \tau_{m e}\left[\left(\ln \frac{N_{c}}{n}\right)+\left(r+\frac{5}{2}\right)\right]}{\frac{p^{2}}{N_{v}} \tau_{m h}+\frac{n^{2}}{N_{c}} \tau_{m e}} .
$$

Compared to the expression derived assuming $\tau_{m} \gg \tau_{m e, m h}$ [Eq. (11) in the paper], (E10) shows a more complex relation to transport properties. It is the bipolar diffusion thermopower. It can be seen that the total thermopower is still not a function of $\tau_{m}$ and is only a weak function of $\tau_{m e, m h}$.

Therefore, for both cases of $\tau_{m} \gg \tau_{m e, m h}$ and $\tau_{m} \ll \tau_{m e, m h}$, the total thermopower of an intrinsic magnetic semiconductor is independent or a weak function of the carriers and magnon relaxation times. 